Tendencias del financiamiento público y dilemas de la política educacional chilena (1999-2018)

Sebastián Donoso-Díaz, Oscar Arias-Rojas y Daniel Reyes-Araya 
Sebastián Donoso-Diaz, Oscar Arias-Rojasy Daniel Reyes-Araya

\section{Tendencias del financiamiento público y dilemas de la política educacional chilena (1999-2018)}

Resumen: Se expone la evolución del presupuesto público cbileno en educación en el presente milenio, atendiendo su relevancia como componente clave del desarrollo, e indagando sobre lo que ello significa en referencia con la evolución del Producto Interno Bruto (PIB) del país, como también del presupuesto público total. Las partidas presupuestarias son actualizadas y analizadas en el contexto de algunos hitos claves de las políticas educativas del periodo estudiado. Los principales resultados muestran incrementos importantes del presupuesto, incluso por sobre el crecimiento del PIB, acordes con las demandas sociales en este campo. No obstante, se estima que deben realizarse algunos exámenes detallados, pues no se puede continuar con un presupuesto sectorial que crece ilimitadamente, sino también se requiere de otras formas de asignación presupuestaria que impliquen un cambio estructural. Los hallazgos evidencian la importancia de avanzar en estas materias para sustentar aquellas políticas estratégicas que Chile no resuelve satisfactoriamente: segmentación social del sistema escolar, desigualdad social y baja calidad de resultados educativos.

Palabras clave: presupuesto público, presupuesto público de educación, educación pública, Ministerio de Educación, Chile

Clasificación JEL: I2, H6, D63, E62.

\section{Trends in Public Financing and Dilemmas of Chilean Educational Policy (1999-2018)}

Abstract: The evolution of the Chilean public budget in education in the present millennium is exposed, attending to its relevance as a key component of development, and inquiring about what this means in reference to the evolution of the country's Gross Domestic Product (GDP), as well as the public budget. -total audience. The annual budget items are systematized, updated, before some key milestones of the educational policies of the period studied. The main results show significant increases in the budget, even above GDP growth, and in the social demands in this field. However, it is estimated that some major examinations must be carried out, since it is not possible to continue with a sector budget that grows unlimitedly, but also to it requires other forms of budget allocation that involve structural change. The findings show the importance of advancing in these matters to support those strategic policies that Chile does not satisfactorily resolve: social segmentation of the school system, social inequality, and low quality of educational results.

Keywords: Public Budget, public education budget, public education, Ministry of Education, Chile

https://doi.org/10.17533/udea.le.n95a343163

\section{(cc) BY-NC-SA}

Este artículo y sus anexos se distribuyen por la revista Lecturas de Economía bajo los términos de la Licencia Creative Commons Atribución-NoComercial-CompartirIgual 4.0. https://creativecommons.org/licenses/by-nc-sa/4.0/ 


\section{Tendances dans le financement public et dilemmes de la politique éducative chilienne (1999-2018)}

Résumé: Nous exposons l'évolution du budget public cbilien de l'éducation au cours du présent millénaire, en tenant compte sa pertinence en tant que composante clé du développement. Nous nous interrogeons sur sa signification par rapport à l'évolution du produit intérieur brut (PIB) du pays, ainsi que par rapport au budget public total. Les allocations budgétaires annuelles sont mises à jour et analysées dans le contexte de certains événements clés des politiques éducatives de la période étudiée. Les principaux résultats montrent des augmentations significatives du budget éducatif, même supérieures à la croissance du PIB, en ligne avec les demandes sociales dans ce secteur. Cependant, nous considérons qu'il faut réviser en détail ces allocations budgétaires, car il est impossible de continuer avec un budget sectoriel qui croît à l'infini. Il y a donc besoin de mettre en place d'autres formes d'allocation budgétaire qui impliquent un changement structurel. Les résultats montrent également l'importance d'avancer dans ces matières, afin de soutenir les politiques stratégiques dont le Chili ne résout pas de manière satisfaisante: segmentation sociale du système scolaire, inégalités sociales et faible qualité des résultats scolaires.

Mots clés: Budget public, budget public en éducation, éducation publique, ministère cbilien de l'education, Chili.

Cómo citar / How to cite this item:

Donoso-Díaz, S., Arias-Rojas, O., \& Reyes-Araya, D. (2021). Tendencias del financiamiento público y dilemas de la política educacional chilena (1999-2018). Lecturas de Economía, (95), 167-197.

https://doi.org/10.17533/udea.le.n95a343163 


\title{
Tendencias del financiamiento público y dilemas de la política educacional chilena (1999-2018)
}

\author{
Sebastián Donoso-Díaz $\oplus^{\mathrm{a}}$, Oscar Arias-Rojas $\oplus^{\mathrm{b}}$ y Daniel \\ Reyes-Araya $\oplus^{\mathrm{c}}$
}

-Introducción. -I. Revisión de literatura. -II. Metodología. -III. Discusión de resultados. -Conclusiones. -Agradecimientos. -Referencias.

Primera versión recibida el 24 de julio de 2020; versión final aceptada el 22 de abril de 2021

\section{Introducción}

Tanto en el pensamiento económico, como en el de carácter social y político de las teorías modernas del desarrollo se sostiene que la educación contribuye decisiva y casi insustituiblemente al desarrollo social. Sus planteamientos fundamentan la prioridad que en la actualidad muchos gobiernos le asignan a la educación y — por tanto- a la importancia de financiarla adecuadamente.

Ello desvela que la educación puede ser una potente palanca de avance de la sociedad y en función de ello, su provisión es hoy una obligación de los Estados como expresión del derecho a educación, por tanto irrenunciable en el nivel escolar (y en casos la superior). Consistente con tal argumentación, es sustantivo conocer cómo y cuánto invierte el Estado en educación, y aunque se trate de una interrogante que no es nueva ni exclusiva para el caso de Chile - es fundamental dar cuenta de ella ya que la política educacional

a Sebastián Donoso-Díaz: profesor titular de la Universidad de Talca, Facultad de Ciencias de la Educación, Instituto de Investigación y Desarrollo Educacional, Chile. Dirección electrónica: sdonoso@utalca.cl https://orcid.org/0000-0002-4744-531X

b Oscar Arias-Rojas: consultor independiente. Dirección electrónica: oshcar.arias@gmail.com https://orcid.org/0000-0003-4468-5446

c Daniel Reyes-Araya: profesor de la Universidad de Talca, Facultad de Ciencias de la Educación, Instituto Investigación y Desarrollo Educacional, Chile. Dirección electrónica: dreyes@utalca.cl https://orcid.org/0000-0003-0593-8033 
Donoso-Díaz, Arias-Rojas y Reyes-Araya: Tendencias del financiamiento público y dilemas...

requiere de un soporte financiero irreemplazable para cumplir los objetivos asignados.

En este marco, todo Estado enfrenta preguntas elementales sobre la escala y naturaleza del financiamiento de la educación obligatoria, debiendo decidir estratégicamente acerca del volumen de recursos que le provisiona y qué iniciativas, políticas y acciones destinará. Huelga decir el rol decisivo del gasto en la instalación y desarrollo de una política pública, y por lo mismo dar cuenta que su evolución es un fenómeno relevante, en el entendido que toda iniciativa presupuestaria posee un trasfondo netamente político.

En el caso chileno, desde los años de 1990 en adelante la educación pasó a tener un lugar destacado en los proyectos políticos en democracia, al contrario del proceso vivido durante la década de los años de 1980, donde el gasto público se redujo en un 24\% real (González, 2003). A partir de 1991 el gasto público en educación se ha triplicado en términos efectivos en las dos primeras décadas de democracia (Marcel \& Tokman, 2005), y desde el año 2014 la serie de leyes de gran impacto financiero aprobadas en el Parlamento, aumentaron las exigencias presupuestarias (Leyes de Inclusión; de Desarrollo Profesional Docente; de Educación Superior, y de Nueva Educación Pública) marcan hitos cruciales en este campo por la gran demanda de recursos frescos para el sector que implican.

Este artículo tiene por finalidad analizar los cambios en el presupuesto y gasto público del Ministerio de Educación de Chile (en adelante MINEDUC), para el período 1999-2018, por la importancia que ha tenido esta materia en el devenir de la política pública sectorial del país (Romaguera \& Gallegos, 2011; Kremerman, 2009), y principalmente por las transformaciones impulsadas a partir de los movimientos estudiantiles de 2006 y 2011, y de las principales iniciativas de políticas públicas implementadas desde el 2014 en materia de gratuidad de la educación superior, limitación del lucro de los propietarios (sostenedores) de establecimientos escolares que reciben subsidio público, nueva institucionalidad de la educación pública escolar subnacional, y otras medidas en este plano que significan un fuerte impacto tendencial en el presupuesto sectorial. 
El análisis de estas materias —a saber, presupuesto nacional y política educacional - es un esfuerzo que explora un campo de estudio aún inicial, donde hay muchas interrogantes, especialmente desde la óptica de la política de financiamiento, por la complejidad técnica y operativa que significa sistematizar la información presupuestaria, siendo una perspectiva relevante para avanzar en una mejor comprensión de las políticas educativas y su sustentabilidad en el tiempo.

El artículo expone — a partir de considerandos teóricos y metodológicos fundamentales para comprender el problema y visión adoptada— la evolución de los ingresos del país contrastándole con la disponibilidad presupuestaria general y —especialmente- del sector educación, referenciando algunos hitos educacionales claves del período, para — finalmente- delinear sus implicancias, siempre en un plano sistémico dada la magnitud y complejidad de la temática abordada.

\section{Revisión de literatura}

Un instrumento clave de toda política educacional es el gasto público del sector —en este caso el correspondiente al MINEDUC - y en esa función, su desagregación en las diversas partidas y programas impulsadas por el ministerio. Por tanto, el gasto del Gobierno Central en educación representa aquellos componentes a los que se asignan los recursos y en función de ello se establecen los factores a los que se destina mayor o menor relevancia, según la provisión realizada (Darville \& Rodríguez, 2007).

El presupuesto es un instrumento fundamental y estratégico del gobierno para el cumplimiento de sus metas, asumido desde la perspectiva de las políticas públicas como el resultado del proceso de negociación política, que tiene por finalidad la generación de iniciativas mediante las cuales se busca incidir en la producción, distribución y consumo de bienes y servicios (Howlett, 2019).

Para fines ilustrativos, en el contexto de la Organización para la Cooperación y el Desarrollo Económico (OCDE), Chile —en los últimos años- es uno de los países con menor proporción de gasto público en 
Donoso-Díaz, Arias-Rojas y Reyes-Araya: Tendencias del financiamiento público y dilemas...

Educación, y por tanto registra uno de los mayores gastos privados en Latinoamérica en educación primaria y secundaria (Cetrángolo \& Curcio, 2017, 2017a). Durante el 2015, en los países de la OCDE el gasto público representó el 91,3\% del gasto en educación escolar, mientras que en Chile -2016- éste fue del $83 \%$ (OCDE, 2017, 2018, MINEDUC, 2018).

Consistentemente, el gasto privado en educación superior chileno supera ampliamente el promedio de OCDE (30\%) y el del resto de los países latinoamericanos, siendo el $60 \%$ del gasto total (Cetrángolo \& Curcio, 2017, 2017a). Específicamente, por cada alumno del sistema primario el Estado chileno destina USD 5000, cifra bajo la media del resto de países de la OCDE, la que se aproxima a USD 8500 por estudiante. Ello se reitera — con una brecha mayor - al considerar el nivel educativo secundario: Chile destina algo más de USD 4900, valor que palidece cuando se compara con los USD 9870 que destina en promedio la OCDE (OCDE, 2018).

De esta manera, el país posee un menor gasto público que el promedio de la OCDE en el sistema escolar y en el superior. En educación básica y media, el porcentaje de gasto privado chileno $(21,2 \%)$ es superado solo por Colombia $(23,4 \%)$. El gasto privado en el sistema terciario nacional prácticamente duplica el promedio de la OCDE, siendo México y Colombia los que se aproximan a Chile con cifras cercanas al 39\%, distante de Argentina donde el $99 \%$ del gasto es público (Rodríguez et al., 2016). En referencia a los procesos educativos, la trayectoria de los resultados de Chile en evaluaciones externas como PISA han aumentado desde la primera participación nacional, tratándose de un referente en el contexto latinoamericano, aunque lejano de los promedios de los países desarrollados más destacados.

El rendimiento de lectura en Chile mejoró desde la primera participación del país en PISA (en 2001) [...] la mayor parte de esa mejora se produjo en el período inicial. Entre 2009 y 2018, no se observaron tendencias significativas en el rendimiento en ninguna asignatura. A pesar de un rendimiento general estable, la proporción de estudiantes con un rendimiento de nivel 5 o superior (mejor desempeño) en lectura creció entre 2009 y 2018 (+1,3\%) y disminuyó en ciencia entre 2006 y 2018 (-0,9\%) (OCDE, 2019, p. 284). 
Estos antecedentes sitúan el caso chileno en el marco que se dio a sí mismo tras integrarse a la OCDE, al revelar las brechas de calidad del sistema y también de gasto que implican no solo que debieran incrementarse los recursos sectoriales y aumentar su eficiencia, sino también responder preguntas de fondo sobre la viabilidad de este camino para el largo plazo y - por tanto- la necesidad de realizar cambios estructurales de mayor envergadura para resolver los problemas educativos que permanecen sin solución satisfactoria —a saber, segmentación social, vulnerabilidad y calidad de la educación (Treviño, 2018; Orellana, 2018)—.

El presupuesto del MINEDUC representa gran parte del gasto del gobierno central en educación, el cual se establece anualmente por ley, previa aprobación parlamentaria. En este se consignan las partidas de gasto y se define los criterios para su ejecución, habilitando al ministerio, con algunas salvaguardas, a reasignar partidas cuando el gasto efectivo sea inferior al presupuestado, pudiendo redistribuirse estos recursos a otros ítems ${ }^{1}$.

Además, es importante analizar esta temática en el marco de la evolución del país, sustentado en una serie de tiempo relevante, de manera de determinar cómo ha variado el presupuesto sectorial frente al presupuesto nacional, como señal de la importancia que adquiere en este contexto, basados en el referente clave de la evolución del Producto Interno Bruto (PIB) y —a partir de ello- comparar la evolución del presupuesto sectorial con la política social —o ministerios del sector- para contextualizar el presupuesto de educación respecto del país, teniendo en cuenta lo señalado por Beyer (2007), que los recursos que se destinan a educación son inferiores a los deseables, y que "el actual sistema de financiamiento de la educación hace muy poco para reconocer las desigualdades"2 (p. 177).

1 La base del sistema presupuestario chileno (Von Gersdorff, 2018) está en la Constitución Política, DL. N¹263/1975, sobre Administración Financiera del Estado, y la Ley $\mathrm{N}^{\circ} 20128 / 2006$ sobre responsabilidad fiscal. El presupuesto nacional se aprueba, ejecuta y evalúa en cuatro fases denominadas Ciclo Presupuestario: Formulación; Discusión y Aprobación; Ejecución, y Evaluación (DIPRES, 2019).

2 Si bien los planteamientos de Beyer son previos a la instalación del Subvención Escolar Preferencial (SEP), que reconoce en parte el factor socioeconómico, lo cierto es que este mecanismo fue evaluado posteriormente como insuficiente (Romaguera \& Gallegos, 2011). 
Donoso-Díaz, Arias-Rojas y Reyes-Araya: Tendencias del financiamiento público y dilemas...

Como señalan Marcel y Tokman (2005): “[... tan importante como el volumen de gasto en educación es su distribución. Esta distribución no es independiente del origen del financiamiento de la educación y de su estructura organizativa" (p. 52). Por lo mismo, en referencia al plano sectorial, se asume que la evolución histórica del gasto comparado en la serie de años releva la importancia de las distintas macro políticas en este plano y su sustentación en el tiempo.

El análisis de estas materias —a saber, presupuesto y gasto del Ministerio de Educación - ha sido insuficientemente estudiado en la literatura chilena (BID, 2018; Gallego \& Sapelli, 2007; Marcel \& Tokman, 2005; Romaguera \& Gallegos, 2011; Villarroel, 2013), sea para determinar el origen de los recursos del sector, o - alternativamente- para indagar sobre los montos asignados a la educación pública; no siempre confrontándolo con la política educacional efectiva, sin asumir el gasto como componente clave de esta última (AMUCH, 2015).

En el plano nacional, el presupuesto público central en educación es la piedra angular de la política educacional de cada gobierno (Raczynski \& Salinas, 2009; Von Gersdorff, 2018). Este gasto sustenta la política gubernamental real, por cuanto los aportes de los municipios -que gestionan en sus comunas la educación pública - son comparativamente de menor significación, orientándose a cubrir partidas deficitarias de las transferencias del gobierno central, además con comportamiento financiero fiscal altamente indisciplinado. Estimaciones indican que —en promedioaproximadamente el 10\% del presupuesto municipal se destina a educación, esencialmente a cubrir gastos corrientes (Raczynski \& Salinas, 2009; Villarroel, 2013), no obstante, este aporte representa cerca del $2 \%$ del gasto público total en educación. De allí la relevancia del análisis de las partidas presupuestarias señaladas. Por tanto, la política educacional impulsada por el ministerio se sustenta en la importancia de ese gasto.

En el ámbito del debate entre visiones estructuralistas como neoliberales del desarrollo socioeconómico, la educación surge como una de las escasas áreas en las que existe un consenso importante sobre su impacto en las políticas públicas, en el crecimiento y equidad social. La realidad de muchos países —incluido Chile_ es que gran parte de la inversión en educación la 
realiza el Estado, financiando de manera directa e indirecta sistemas públicos y privados de enseñanza (OCDE, 2017, 2017a, 2018).

Paralelamente, la educación también es una materia social y económica prioritaria para los ciudadanos, no solamente por lo señalado, sino además por las tasas de rentabilidad — social y privada - que aún muestran signos positivos. Cuestión que en Chile es sustantiva y explica parte de las tensiones entre los procesos privatizadores de los años de 1980 y 1990, como también de la reversión de la privatización experimentada desde el año 2015 a la fecha, que involucran tanto a la educación escolar, con la implementación de los nuevos Servicios Locales de Educación Pública - 2018- y por el incremento progresivo de la gratuidad de los aranceles de los estudiantes en la educación superior desde el 2016 (DIPRES, 2018).

Chile superó formalmente las metas de cobertura universal de la educación escolar (excepto en el nivel parvulario), lo que implica nuevos objetivos, uno de los cuales es mejorar la calidad de la enseñanza.

Como la calidad es la dimensión del desempeño educativo que se supone más directamente vinculada a la gestión de los establecimientos educacionales, la discusión sobre cómo mejorar la calidad de la educación en Chile se ha centrado crecientemente en la gestión. Así, se ha llegado a asumir que mejorar la gestión educativa es una condición necesaria -y para algunos incluso suficiente- para elevar la calidad y equidad de la educación que reciben los niños y jóvenes chilenos (Marcel, 2009, p. 35).

De esta forma, el financiamiento de la educación se ha posicionado como factor estratégico. No obstante, aunque actualmente parezca difícil de comprender, en Chile hay escasos referentes empíricos validados respecto de los costos reales de la provisión de educación de calidad que se espera, en diferentes contextos y condiciones socioeconómicas de su población. Lo señalado se evidencia en las conclusiones del Consejo Asesor Presidencial (2006), al establecer que "el Consejo quiere aclarar que hoy no se cuenta con estimaciones de costos que tomen en cuenta los nuevos estándares de calidad, y que asuman todas las diferencias que deben considerarse para estos efectos" (Consejo Asesor Presidencial para la Calidad de la Educación, 2006, p. 129). Estimaciones que más de una década después aún son frágiles. 
Donoso-Díaz, Arias-Rojas y Reyes-Araya: Tendencias del financiamiento público y dilemas...

Una condición esencial para que los establecimientos educacionales puedan alcanzar estándares de calidad más altos, es que el sistema de financiamiento reconozca debidamente las diferencias de costos de los distintos niveles (preescolar, básica, media), modalidades (científico humanista y técnico profesional), localizaciones (ruralidad, aislamiento geográfico y densidad poblacional) y características de la población atendida (nivel socioeconómico, necesidades educativas especiales, condiciones étnicas y de migración). Siendo necesario que los montos que se transfieran a las instituciones correspondan a los parámetros expuestos, "en que se reconozca explícitamente la relación entre el logro de ciertos niveles de calidad y los recursos que, administrados en forma eficiente, permitirían alcanzar dichos niveles" (Consejo Asesor Presidencial para la Calidad de la Educación, 2006, p. 134).

Desde una perspectiva complementaria, parte relevante de los problemas distributivos tienen su origen en la desigualdad de oportunidades al inicio de la trayectoria educativa, que a su vez impacta de manera significativa en las posibilidades de lograr niveles más elevados de productividad, desarrollo y empleo de calidad (PNUD, 2017). Debido a ello, existen presiones sociales por incrementar la eficiencia del gasto en educación como requisito — la mayoría de las veces- del aumento del financiamiento del sector, para contribuir al mejoramiento de la calidad y la equidad, sin resentir el alcance y la cobertura del sistema escolar (Hanushek \& Woessmann, 2015).

De lo expuesto surge como factor decisivo los recursos públicos asignados a educación, que se expresa en el presupuesto público nacional del sector - asignado al Ministerio de Educación- como instrumento de soporte de las políticas educacionales. En este mecanismo público de política (el presupuesto del país) se combinan los recursos destinados a la operación regular del sistema con las iniciativas específicas de intervención que cada gobierno define y trasunta en su propuesta presupuestaria. Razón importante para sustentar la relevancia de estudiar la asignación de recursos públicos al MINEDUC, y su función como soporte de las políticas —analizando una extensa serie temporal—, y su incidencia en las políticas educativas. 
Entendiendo que las reformas educativas de la actual generación se centran en los incrementos relevantes de la calidad de la educación —materia en la cual el país pese a los progresos registrados está lejos de los logros de países del primer mundo- y habiendo otros territorios que han realizado avances muy acelerados —incluso bajo condiciones sociales y económicas más precarias que Chile_-, se deduce que, con alta seguridad, las reformas requerirán de mayores recursos. Pero no siempre estos incrementos se traducirán en mejores resultados educativos, por lo cual este desafío es mucho más complejo. Para determinar nuevos formatos presupuestarios, se hace necesario disponer de información sistemática para adoptar las decisiones estratégicas en esta materia que consideren —incluso— cambios estructurales en el financiamiento de la educación.

\section{Metodología}

En este artículo, se analizan los presupuestos nacionales chilenos del período 1999-2018, a partir de los informes de la Dirección de Presupuestos (DIPRES). Se considera el presupuesto aprobado por el Congreso para el año correspondiente. Se incorporó el presupuesto en pesos (actualizado a diciembre de 2018).

Las clasificaciones del presupuesto público ${ }^{3}$ se aplican a todos los organismos y establecen la necesidad de desagregar y definir el contenido de los conceptos de ingresos y gastos a observarse en la ejecución presupuestaria e información pertinente ${ }^{4}$, manteniendo una estructura permanente. Según Blöndal y Curristine (2004 en Von Gersdorff, 2018) esta se puede ordenar de distintas maneras por el objetivo, esto es, por categoría, institucional y objeto. Para las comparaciones sectoriales (ministeriales) se emplean las agrupaciones presentadas en la Tabla 1.

3 El clasificador presupuestario corresponde al Decreto del Ministerio de Hacienda ( $\mathrm{N}^{\circ} 854 / 2004$ y modificaciones).

4 El gasto público, identifica los recursos que desembolsa el gobierno en la compra de bienes y servicios, en los pagos previsionales, en el pago de intereses, en las transferencias y sus inversiones, a partir del presupuesto aprobado vía Ley de Presupuestos y sus modificaciones en el período contable siguiente. 
Donoso-Díaz, Arias-Rojas y Reyes-Araya: Tendencias del financiamiento público y dilemas...

Tabla 1. Comparaciones sectoriales ministeriales

\begin{tabular}{|c|c|}
\hline Trabajo y Previsión Social & $\begin{array}{l}\text { Ministerio del Trabajo y Previsión Social Fondo de } \\
\text { Reserva de Pensiones }\end{array}$ \\
\hline Educación & Ministerio de Educación Fondo de Educación \\
\hline Defensa & Ministerio de Defensa Nacional \\
\hline Economía, Finanzas, Desarrollo y Cultura & $\begin{array}{l}\text { Ministerio de Hacienda Ministerio de Economía } \\
\text { Ministerio de Obras Públicas Ministerio de } \\
\text { Agricultura Ministerio de Minería Ministerio de } \\
\text { Transporte y Telecomunicaciones Ministerio de } \\
\text { Desarrollo Social Ministerio de Energía Ministerio } \\
\text { del Medio Ambiente Ministerio del Deporte } \\
\text { Ministerio de la Mujer y la Equidad de Género } \\
\text { Ministerio de las Culturas }\end{array}$ \\
\hline Políticos, Administrativos y Justicia & $\begin{array}{l}\text { Presidencia de la República Congreso Nacional } \\
\text { Poder Judicial Contraloría General de la República } \\
\text { Ministerio del Interior Ministerio de Relaciones } \\
\text { Exteriores Ministerio de Justicia y Derechos } \\
\text { Humanos Ministerio de Bienes Nacionales } \\
\text { Ministerio Secretaria General de Gobierno } \\
\text { Ministerio Secretaria General de la Presidencia } \\
\text { Ministerio Público Servicio Electoral Fondo de } \\
\text { Apoyo Regional }\end{array}$ \\
\hline Salud & $\begin{array}{l}\text { Ministerio de Salud Fondo Tratamientos de Alto } \\
\text { Costo }\end{array}$ \\
\hline Vivienda & Ministerio de Vivienda y Urbanismo \\
\hline Servicio de la Deuda & Servicio de la Deuda Pública \\
\hline Operaciones Complementarias & Operaciones Complementarias \\
\hline Otros & $\begin{array}{l}\text { Subsidios Fondo de Estabilización Económica y } \\
\text { Social }\end{array}$ \\
\hline
\end{tabular}

Fuente: elaboración propia a partir de Blöndal y Curristine (2004). 
Por otro lado, se consideró la información del PIB nominal, real y deflactores provenientes del Banco Central de Chile para cada período. Adicionalmente, la información del gasto municipal en educación se obtuvo de los informes financieros municipales de la Contraloría General de la República. Para hacer comparables los datos de los distintos informes de esa institución se contempló el gasto anual devengado en educación y, en este ítem, los aportes municipales a la educación pública comunal.

Todas las cifras se expresan en valores del año 2018 para efecto del análisis y sus comparaciones. Los años incluidos poseen datos completos y consistentes, su selección responde a este criterio y a la potencialidad que entrega una serie de dos décadas para la solidez de su análisis.

\section{Discusión de resultados}

\section{A. Panorama del presupuesto público en educación}

En cuanto a las dos décadas de estudio (1999-2018), el presupuesto del MINEDUC —en términos efectivos - se ha cuadruplicado, aumentando desde un total de CLP M\$2404756717 en 1999 a CLP M\$10031615939 en 2018 (Tabla 2), creciendo un promedio anual de 7,8\% en esos años. En consecuencia, se registra una tendencia de aumento importante del presupuesto sectorial, respondiendo a la universalización de la educación escolar primaria y secundaria (la básica había sido lograda) y también de grupos sociales medios por el acceso a la educación superior, expresados además por instituciones no gubernamentales, gremios y partidos políticos.

El presupuesto del MINEDUC no es el único gasto público en educación en Chile. La Tabla 2 muestra tanto su evolución, como la de dos fuentes públicas adicionales: primero, el aporte municipal a la educación pública; segundo, aportes de otras partidas de gasto que se incorporan al presupuesto nacional, pero que no se asignan mediante el MINEDUC. 
Donoso-Díaz, Arias-Rojas y Reyes-Araya: Tendencias del financiamiento público y dilemas...

Tabla 2. Aportes públicos a educación. Chile, 1999-2018

\begin{tabular}{|c|c|c|c|c|c|c|c|}
\hline \multirow[t]{2}{*}{ Años } & \multicolumn{2}{|c|}{$\begin{array}{l}\text { Presupuesto } \\
\text { Ministerio de } \\
\text { Educación }\end{array}$} & \multicolumn{2}{|c|}{ Aporte Municipal } & \multicolumn{2}{|c|}{$\begin{array}{c}\text { Otros aportes } \\
\text { públicos a educación } \\
\text { en el presupuesto de } \\
\text { la Nación }\end{array}$} & \multirow[t]{2}{*}{ Total (CLP M) } \\
\hline & $\%$ & CLP M & $\%$ & CLP M & $\%$ & CLP M & \\
\hline 1999 & $97,5 \%$ & 2404756717 & $2,5 \%$ & 60619354 & $0,0 \%$ & & 2465376072 \\
\hline 2000 & $97,6 \%$ & 2561839000 & $2,4 \%$ & 63693249 & $0,0 \%$ & & 2625532249 \\
\hline 2001 & $97,5 \%$ & 2738636793 & $2,5 \%$ & 70984315 & $0,0 \%$ & & 2809621108 \\
\hline 2002 & $97,6 \%$ & 3055691924 & $2,4 \%$ & 74462812 & $0,0 \%$ & & 3130154736 \\
\hline 2003 & $97,6 \%$ & 3207589349 & $2,4 \%$ & 78051977 & $0,0 \%$ & & 3285641326 \\
\hline 2004 & $97,3 \%$ & 3333108571 & $2,7 \%$ & 92018084 & $0,0 \%$ & & 3425126655 \\
\hline 2005 & $97,2 \%$ & 3425738738 & $2,8 \%$ & 98389176 & $0,0 \%$ & & 3524127914 \\
\hline 2006 & $97,1 \%$ & 3666157522 & $2,9 \%$ & 108672321 & $0,0 \%$ & & 3774829843 \\
\hline 2007 & $97,2 \%$ & 3896768861 & $2,8 \%$ & 113003408 & $0,0 \%$ & & 4009772269 \\
\hline 2008 & $98,2 \%$ & 4576534656 & $1,8 \%$ & 83830436 & $0,0 \%$ & & 4660365092 \\
\hline 2009 & $98,1 \%$ & 5328117768 & $1,9 \%$ & 103242023 & $0,0 \%$ & & 5431359791 \\
\hline 2010 & $98,2 \%$ & 5931103843 & $1,8 \%$ & 108380742 & $0,0 \%$ & & 6039484585 \\
\hline 2011 & $98,1 \%$ & 6458272423 & $1,9 \%$ & 122037815 & $0,0 \%$ & & 6580310237 \\
\hline 2012 & $98,0 \%$ & 7064729066 & $2,0 \%$ & 141060834 & $0,0 \%$ & 2517 & 7205792417 \\
\hline 2013 & $76,3 \%$ & 7758468590 & $1,4 \%$ & 144873090 & $22,3 \%$ & 2262407005 & 10165748684 \\
\hline 2014 & $93,7 \%$ & 7709811654 & $1,7 \%$ & 143705499 & $4,5 \%$ & 373574182 & 8227091334 \\
\hline 2015 & $94,1 \%$ & 8356382788 & $1,5 \%$ & 131362912 & $4,4 \%$ & 388101332 & 8875847032 \\
\hline 2016 & $89,3 \%$ & 9156331317 & $1,2 \%$ & 123668293 & $9,5 \%$ & 972513426 & 10252513036 \\
\hline 2017 & $86,0 \%$ & 9501986815 & $1,1 \%$ & 122928394 & $12,8 \%$ & 1418481068 & 11043396276 \\
\hline 2018 & $87,6 \%$ & 10031615939 & $1,0 \%$ & 113914995 & $11,4 \%$ & 1303150619 & 11448681553 \\
\hline
\end{tabular}

Nota: el aporte municipal se estimó utilizando el porcentaje del PIB que representó el aporte municipal en 2000. Miles de pesos de 2018.

Fuente: DIPRES (2014, 2017, 2018, 2019) y Contraloría General de la República (2018). 
Con relación al aporte municipal a educación, este responde al modelo de municipalización de la educación pública instalado desde $1981^{5}$, que administra la educación pública escolar, entes de comprometer financiamiento público adicional al provisto desde el nivel central. Este financiamiento deriva de recursos de los gobiernos locales (Villarroel, 2013), generados por tributos y otras transferencias desde el nivel central ${ }^{6}$. Este gasto representa en promedio el $10 \%$ del gasto total municipal (Raczynski \& Salinas, 2009). No obstante, comparado con el gasto del MINEDUC, los recursos comunales tienen un impacto bastante menor, no superando el $3 \%$ del gasto ministerial, debido a que las partidas del MINEDUC no solamente consideran la educación escolar pública, sino también la educación superior y los aportes a las escuelas privadas que operan con subvención del Estado. Para el año 2018, el aporte municipal a la educación representó solo un 1,1\% del total del presupuesto de educación, producto del aumento del gasto a nivel central del ministerio y de la disminución de los aportes totales municipales.

El gasto municipal en educación ha sido debidamente estudiado, correspondiendo por lo general a consumos corrientes — remuneraciones, mantenimiento de infraestructura y otras-, más que a implantación de políticas educativas específicas de carácter local por el municipio (Raczynski \& Salinas, 2009).

Por otro lado, desde 2013 se incluyen en el presupuesto nacional recursos destinados a educación, los que no se contemplan en el presupuesto inicial del MINEDUC. Estos corresponden al Fondo para la Educación, creado por la Ley 20630 que perfecciona la legislación tributaria y financia la reforma educacional. El artículo 7 de esa ley establece:

Créase un Fondo Especial para la Educación, [...] destinado a complementar el financiamiento de las obligaciones fiscales

5 En el 2018 se inicia un proceso gradual de desmunicipalización de la educación escolar pública, con la instalación de los Servicios Locales de Educación. Durante el 2018 se implementaron los primeros cuatro servicios, el 2019 tres, y cuatro en 2020, de un total de 70 .

6 El gasto municipal en educación no incluye trasferencias y subvenciones específicas recibidas desde el nivel central. 
Donoso-Díaz, Arias-Rojas y Reyes-Araya: Tendencias del financiamiento público y dilemas...

contenidas en la Partida del Ministerio de Educación de la Ley de Presupuestos del Sector Público. Serán usos preferentes de los recursos del Fondo para la Educación, los destinados a educación pre-escolar, subvención escolar preferencial y becas y créditos para educación superior.

Este Fondo se origina el 2013 con una inversión inicial de USD 4000 millones proveniente de la liquidación del Fondo de Estabilización Económica y Social ${ }^{7}$, y se dirige a financiar la reforma en educación preescolar, aumento de subvención escolar y financiamiento de la educación superior (algunos de los cuales se derivan de las demandas del movimiento estudiantil del año 2011). Estos recursos no fueron incluidos en el presupuesto ministerial ya que estarían disponibles solo a partir de 2014, pues durante 2013 el gobierno central debió realizar las operaciones para completar este fondo. De este modo, esos dineros no aparecen en la ley en el registro contable de ingresos del MINEDUC del año correspondiente. Sin embargo, se realizan transferencias desde este fondo a los programas del ministerio en el año siguiente, cumpliendo así su función (DIPRES, 2014).

\section{B. Crecimiento del PIB, gasto público total y presupuesto de educación}

Al considerar el presupuesto anual total del país en el período 19992018, se observa un incremento real de 356\%, desde CLP MM $\$ 13009480$ en 1999 a CLP MM\$46865211 en el 2018, correspondiendo a un aumento anual real promedio de 7,0\% (Tabla 3). En este marco es importante destacar que el aumento efectivo del presupuesto de educación ha sido superior al del presupuesto total del país. El incremento presupuestario promedio anual de educación alcanza a 7,8\% en los años considerados, casi un $1 \%$ anual, y considerando el total de los recursos destinados al MINEDUC y el Fondo de Educación, éste llega al 8,5\% promedio anual, valor relevante de lo que representan estos recursos para la tarea educativa - aproximadamente, CLP M\$300 por año (USD 420000)—.

Fondo que reúne recursos excedentarios del país, invertidos en instrumentos de gobiernos y

fondos internacionales. 
Tabla 3. Presupuesto público y presupuesto en educación como proporción del PIB. Chile, $1999-2018$

\begin{tabular}{cccccccc}
\hline \multirow{2}{*}{ Años } & \multicolumn{2}{c}{$\begin{array}{c}\text { Presupuesto público } \\
\text { Total }\end{array}$} & \multicolumn{2}{c}{$\begin{array}{c}\text { Presupuesto } \\
\text { MINEDUC }\end{array}$} & $\begin{array}{c}\text { Presupuesto MINEDUC } \\
\text { + Fondo de Educación }\end{array}$ & PIB total \\
\cline { 2 - 7 } & CLP M & \% del PIB & CLP M & \% del PIB & CLP M & \% del PIB & \\
\hline $\mathbf{1 9 9 9}$ & 13009480025 & 18,6 & 2404756717 & 3,4 & & 69977493850 \\
$\mathbf{2 0 0 0}$ & 13489706064 & 18,3 & 2561839000 & 3,5 & & 73525922276 \\
$\mathbf{2 0 0 1}$ & 14.800587072 & 19,3 & 2738636793 & 3,6 & & 76861103616 \\
$\mathbf{2 0 0 2}$ & 15164 & 19,5 & 3055691924 & 3,8 & & 79686736431 \\
$\mathbf{2 0 0 3}$ & 16413471427 & 19,1 & 3207589349 & 3,7 & & 85824079721 \\
$\mathbf{2 0 0 4}$ & 16779628278 & 17,3 & 3333108571 & 3,4 & & & 96881414639 \\
$\mathbf{2 0 0 5}$ & 18278937196 & 17,2 & 3425738738 & 3,2 & & & 1063776693881 \\
$\mathbf{2 0 0 6}$ & 20554158122 & 16,6 & 3666157522 & 3,0 & & 123677271200 \\
$\mathbf{2 0 0 7}$ & 24940929490 & 19,7 & 3896768861 & 3,1 & & 126753679968 \\
$\mathbf{2 0 0 8}$ & 26339881958 & 21,5 & 4576534656 & 3,7 & & & 122470810948 \\
$\mathbf{2 0 0 9}$ & 30870312107 & 24,1 & 5328117768 & 4,2 & & & 127932092483 \\
$\mathbf{2 0 1 0}$ & 30128241720 & 21,0 & 5931103843 & 4,1 & & & 143268563499 \\
$\mathbf{2 0 1 1}$ & 31544781677 & 21,0 & 6458272423 & 4,3 & & & 150106270578 \\
$\mathbf{2 0 1 2}$ & 33952372036 & 21,6 & 7064729066 & 4,5 & & & 157527108272 \\
$\mathbf{2 0 1 3}$ & 39315767138 & 24,2 & 7758468590 & 4,8 & 10020875595 & 6,2 & 162252833897 \\
$\mathbf{2 0 1 4}$ & 36104522786 & 21,6 & 7709811654 & 4,6 & 8083385835 & 4,8 & 167095519977 \\
$\mathbf{2 0 1 5}$ & 39114331025 & 22,8 & 8356382788 & 4,9 & 8744484120 & 5,1 & 171902890203 \\
$\mathbf{2 0 1 6}$ & 42204314479 & 23,7 & 9156331317 & 5,2 & 10128844743 & 5,7 & 1777716404333 \\
$\mathbf{2 0 1 7}$ & 44270127229 & 24,0 & 9501986815 & 5,1 & 10920467883 & 5,9 & 184839626963 \\
$\mathbf{2 0 1 8}$ & 46865211788 & 24,5 & 10031615939 & 5,2 & 11334766558 & 5,9 & 191248710869 \\
\hline
\end{tabular}

Nota: miles de pesos de 2018.

Fuente: DIPRES (2014, 2017, 2018, 2019) y Banco Central de Chile (2018).

En los años estudiados se registra un aumento promedio anual real del PIB de 5,4\%, pasando en 1999 de MM CLP 69977493 a MM CLP 191248710 en 2018 (aumentó 2,7 veces). No obstante, el presupuesto en educación lo hace 4,7 veces, superando el crecimiento del PIB del período, tanto en los presupuestos de educación como en el presupuesto total de la nación. La prioridad que educación para el país se refrenda al constatar: 
Donoso-Díaz, Arias-Rojas y Reyes-Araya: Tendencias del financiamiento público y dilemas...

Una medida básica de la prioridad que reviste la educación dentro de una sociedad es estimar qué proporción del PIB se destina a la inversión educativa (...) Dakar puso énfasis en la necesidad de que los gobiernos expandieran su compromiso financiero con la educación. En esta materia, en promedio, los países de América Latina y el Caribe aumentaron el nivel de gasto público en educación como porcentaje del PIB, pasando de 4,5 en 2000 a 5,2 en 2010, es decir, acumulando un aumento de siete décimas porcentuales, y acercándose al promedio de gasto en educación de los países europeos y Estados Unidos, que hacia 2010 era de 5,6 \% del PIB (UNESCO, 2013, p. 37).

Ello evidencia el esfuerzo fiscal chileno, que vino acompañado de gran inversión en seguridad pública y de una reforma judicial, en momentos que se atravesaba por demandas sociales que fueron cruciales respecto al modelo de sociedad impuesto en dictadura. De este modo, mientras que en 1999 el país destinó a educación un presupuesto equivalente al 3,4 \% del PIB, en 2018 éste fue de $5,9 \%$, sin considerar que la participación de los recursos privados en el gasto total en educación es prácticamente del 35 a $40 \%$ del aporte total (OCDE, 2017, 2018). En un marco en que el país registra un aumento en el presupuesto general que en 1999 supuso un 18,6\% del producto, en tanto que en 2018 fue del 24,5\% del PIB.

\section{Tendencias del presupuesto público de educación}

Acerca de lo que representa el presupuesto de educación contrastado con el gasto público total (Figura 1), analizada la composición de los presupuestos nacionales del mismo período, se observa una disminución en el presupuesto del Ministerio del Trabajo y Previsión Social y — como contrapartida- el aumento de Educación, con énfasis desde el $2007^{8}$. También, el gasto en defensa como proporción del presupuesto nacional disminuye, especialmente

8 Analizando detalladamente el presupuesto, no es casualidad que el movimiento estudiantil de mayo de 2006, planteara fuertes exigencias de mejoramiento en infraestructura y equipamiento de los centros escolares públicos, así como cambios normativos que ciertamente impactaron en esta materia. 
con posterioridad al 2011; también se registra en ese año un leve aumento del gasto proporcional en salud.

Figura 1. Presupuestos de educación en el contexto del gasto público sectorial

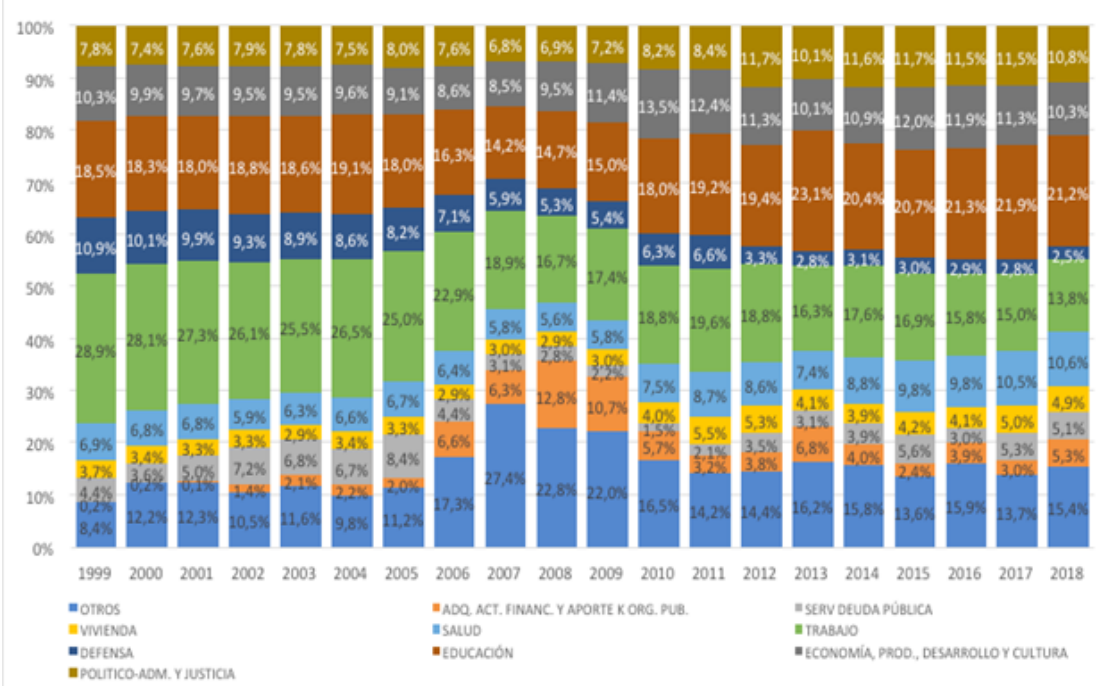

Fuente: DIPRES (2014, 2017, 2018, 2019).

Un examen detallado del gasto como proporción del PIB por sector (Figura 2) muestra para período 2003-2006 un menor crecimiento relativo del gasto en educación y también entre los años 2002 a 2006 en trabajo y previsión social. A partir de los presupuestos de 2007 y 2008 se asigna una mayor proporción de recursos educación y trabajo, respectivamente. Parte de ello es por las medidas implementadas por el gobierno como respuestas al movimiento del 2006 que demandó cambios sustantivos en educación pública en materia normativa, institucional y financiera.

A partir de 2012, tras el movimiento estudiantil del año anterior, el gasto en educación como proporción del PIB continuó incrementándose. Con posterioridad al 2013, el mayor gasto del presupuesto nacional corresponde a este rubro. Como se señaló, en el presupuesto conjunto del MINEDUC y del Fondo de Educación del 2018, equivale al 5,9\% del PIB, mientras que al inicio de la serie analizada (1999) el presupuesto total del sector era del 3,4\%. 
Donoso-Díaz, Arias-Rojas y Reyes-Araya: Tendencias del financiamiento público y dilemas...

Los resultados reseñados en la Figura 2 evidencian que, con cierta regularidad, la distribución presupuestaria varía anualmente, sea por la intencionalidad política de los gobiernos de turno, como por demandas sociales y presiones de grupos de poder. En este escenario, el presupuesto de educación ha crecido en términos absolutos y relativos, acusando recibo a las demandas de mayor escolaridad y calidad de la enseñanza —estas últimas aun marcadamente insatisfechas - y de su intencionalidad política, por ende el aumento de los recursos necesita dialogar con la efectividad de las políticas para avanzar a un diseño que apunte mejor a los objetivos sustantivos sectoriales.

Figura 2. Principales sectores en el presupuesto nacional como proporción del PIB. Chile, 1999-2018

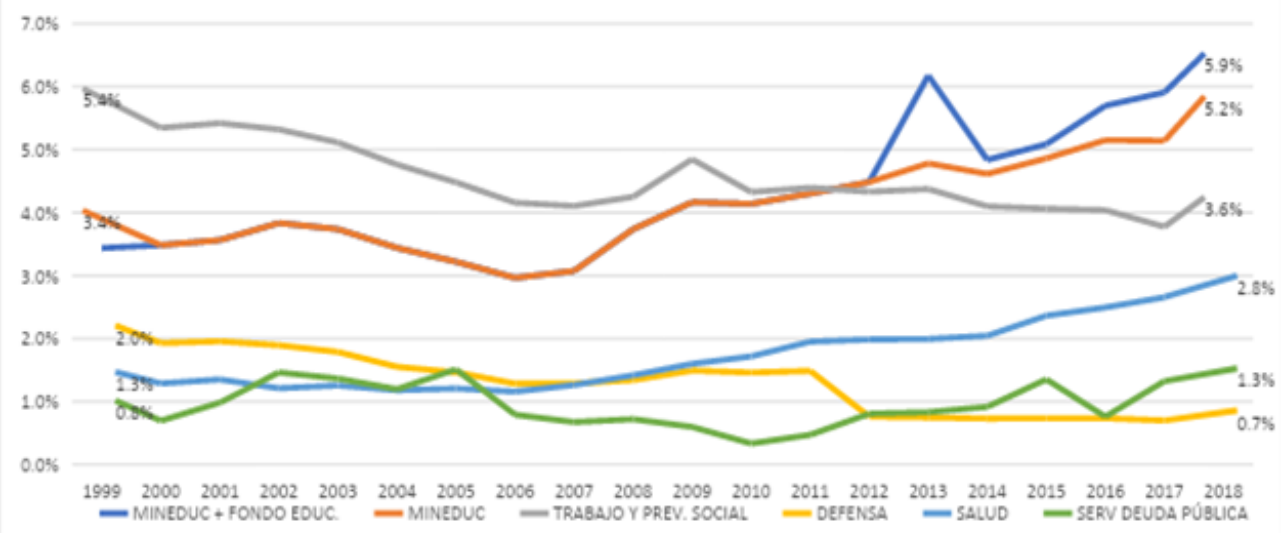

Fuente: DIPRES (2014, 2017, 2018, 2019) y Banco Central de Chile (2018).

Finalmente, se analizan las dinámicas del gasto en educación en los gobiernos del período 2000-2018. Si bien cada gobierno inicia su etapa con un presupuesto aprobado en la administración anterior, se asume ese dato como factor dado. En estas dos décadas se enmarcan cuatro presidencias completas: Ricardo Lagos (2000-2005), Michelle Bachelet (2006-2009), Sebastián Piñera (2010-2013) y Michelle Bachelet (2014-2017). Esta información se encuentra registrada en la Tabla 4. 
Tabla 4. Gasto público, Gasto en Educación, crecimiento del PIB y gobiernos en el periodo. Chile, 2000-2018

\begin{tabular}{|c|c|c|c|c|c|c|c|c|}
\hline \multirow{2}{*}{$\begin{array}{l}\text { Presidente } \\
\text { (a) }\end{array}$} & \multirow{2}{*}{ Período } & \multicolumn{2}{|c|}{$\begin{array}{l}\text { Presupuesto público } \\
\text { total }\end{array}$} & \multicolumn{2}{|c|}{$\begin{array}{l}\text { Presupuesto } \\
\text { MINEDUC }\end{array}$} & \multicolumn{2}{|c|}{$\begin{array}{l}\text { Presupuesto MINEDUC } \\
\text { + Fondo de Educación }\end{array}$} & \multirow{2}{*}{$\begin{array}{l}\text { PIB total } \\
\text { CLP MM }\end{array}$} \\
\hline & & CLP MM & $\%$ del PIB & CLP MM & \% del PIB & CLP MM & $\%$ del PIB & \\
\hline \multirow{5}{*}{$\begin{array}{l}\text { Ricardo } \\
\text { Lagos E. }\end{array}$} & 2000 & 13489706 & 18,3 & 2561839 & 3,5 & & & 73525922 \\
\hline & 2005 & 18278937 & 17,2 & 3425738 & 3,2 & & & 106377669 \\
\hline & Dif CLP & 4789231 & $-1,1$ & 863899 & $-0,3$ & & & 32851747 \\
\hline & Dif \% & 35,5 & & 33,7 & & & & 44,6 \\
\hline & Prop. & 1,36 & & 1,30 & & & & 1,45 \\
\hline \multirow{5}{*}{$\begin{array}{l}\text { Michelle } \\
\text { Bachelet J. }\end{array}$} & 2006 & 20554158 & 16,6 & 3666157 & 3,0 & & & 123677271 \\
\hline & 2009 & 30870312 & 24,1 & 5328117 & 4,2 & & & 127932092 \\
\hline & Dif CLP & 10326154 & 7,5 & 1661960 & 1,2 & & & 4254821 \\
\hline & Dif $\%$ & 50,2 & & 41,5 & & & & 3,4 \\
\hline & Prop. & 1,50 & & 1,41 & & & & 1,03 \\
\hline \multirow{5}{*}{$\begin{array}{l}\text { Sebastián } \\
\text { Pińera E. }\end{array}$} & 2010 & 30128241 & 21,0 & 5931103 & 4,1 & 5931103 & 4,1 & 143268563 \\
\hline & 2013 & 39315767 & 24,2 & 7758468 & 4,8 & 10020875 & 6,2 & 162252833 \\
\hline & Dif CLP & 9187526 & 3,2 & 1827365 & 0,7 & 4089772 & 2,2 & 18984270 \\
\hline & Dif \% & 30,5 & & 30,8 & & 69 & & 13,3 \\
\hline & Prop. & 1,30 & & 1,30 & & 1,70 & & 1,10 \\
\hline \multirow{5}{*}{$\begin{array}{l}\text { Michelle } \\
\text { Bachelet J. }\end{array}$} & 2014 & 36104522 & 21,6 & 7709811 & 4,6 & 8083385 & 4,8 & 167095519 \\
\hline & 2017 & 44270127 & 24,0 & 9501986 & 5,1 & 10920467 & 5,9 & 184839626 \\
\hline & Dif CLP & 8165605 & 2,4 & 1792175 & 0,5 & 2837082 & 2,1 & 17744107 \\
\hline & Dif \% & 22,6 & & 23,2 & & 35,1 & & 106 \\
\hline & Prop. & 1,22 & & 1,23 & & 1,35 & & 1,10 \\
\hline
\end{tabular}

Fuente: DIPRES $(2014,2017,2018,2019)$ y Banco Central de Chile (2018).

En este caso, un elemento central del análisis a nivel internacional da cuenta para América Latina y el Caribe que no es menester solo el comparativo respecto a la relación del gasto en educación y PIB. Si pretendemos establecer una referencia razonada, es dable contemplar la proporcionalidad del Estado en una economía local y el gasto educativo respecto al total como eje de esa prioridad (educación). Por ello:

Dado que los países difieren significativamente en el tamaño proporcional del Estado respecto a la economía nacional, un indicador más ajustado para evaluar la prioridad financiera que los 
Donoso-Díaz, Arias-Rojas y Reyes-Araya: Tendencias del financiamiento público y dilemas...

gobiernos dan a la educación es comparar el gasto en este sector con el gasto público total. En esta materia, en general, los países de la región no mostraron una tendencia positiva durante la década anterior, por cuanto el promedio del gasto público en educación como porcentaje del gasto del gobierno disminuyó levemente, desde un $14,8 \%$ en 2000 a un 14,1\% en 2010 (UNESCO, 2013, p. 38).

Para Chile, esta relación es favorable y se puede desglosar en la Tabla 4, que compara datos de inicio y término de cada período presidencial para conformar una síntesis de su devenir, tanto respecto de la variación del PIB total, del presupuesto público total y del correspondiente al MINEDUC, el cual incluye el fondo adicional que empezó a operar el 2013. Para las comparaciones relativas se emplea el año inicial del período, como situación basal.

Los resultados de la Tabla 4 muestran algunas situaciones que ameritan un estudio más detallado a partir de los antecedentes que se aportan en el presente artículo. Primero, en el gobierno de Lagos el PIB nacional incrementa sustantivamente su valor, en tanto en el presupuesto nacional general y el sectorial de educación crece por debajo de ello. Es significativo este punto, pues el crecimiento PIB podría haber permitido un mayor sustento del gasto en educación. Sin embargo, el país atendió otras materias de seguridad y administración de justicia que también eran importantes.

En los dos gobiernos de Michelle Bachelet el sector educación se expande a tasas superiores al PIB, similares al incremento presupuestario del país. Lo que en ambos gobiernos es de mayor peso relativo por la menor expansión del PIB, exponiendo que el avance de educación en este campo reviste una intencionalidad política más evidente, también porque los movimientos estudiantiles la habían instalado en la agenda pública como prioridad mayor.

En el Gobierno de Sebastián Piñera — de tendencia liberal en lo económico-, esta expansión corresponde esencialmente al fondo de educación que fue impulsado en el marco de las soluciones al movimiento estudiantil de 2011 y que tampoco resolvió los temas sustantivos planteados. 


\section{Conclusiones}

El análisis del presupuesto público de educación expone materias que requieren mayor estudio y profundización, campo aún en ciernes, que de todas formas son un aporte para el análisis de las macro políticas educativas impulsadas. Si bien es factible determinar cuestiones generales sobre cuáles fueron las políticas que se beneficiaron de los incrementos - fortalecimiento de la educación escolar pública y gratuidad de educación superior, entre otras - y cuáles por los decrementos — como la instalación en el 2008 del subsidio escolar complementario por vulnerabilidad (SEP)—, o de nuevas modalidades de crédito y becas para estudiantes de educación superior, o de la gratuidad en educación superior desde el 2016 y de los nuevos servicios locales de educación pública el año 2018.

El presupuesto de educación entre los años 1999 y 2018 crece del 3,4\% al 5,9\% del PIB, siendo importante en el plano político, financiero y social. El presupuesto como porcentaje del PIB tiene su menor expresión en la serie de años del 2001-2005, con especial atención que en ese lapso el país registró mayor crecimiento anual del PIB que en los años siguientes, lo que modera su impacto absoluto, pero nos interroga acerca de porqué no se incrementó en mayor grado su participación dado los problemas de arrastre de la educación en cuestiones claves de calidad de la enseñanza (infraestructura escolar, equipamiento, etcétera), desigualdad y segregación social. Adicionalmente, a partir de 2007 el presupuesto sectorial como parte del PIB se eleva en 1,2\% promedio anual, y en el período final bajo estudio — 2014 en adelante- en $1,8 \%$.

La educación ha sido un área sustantiva de atención financiera del Estado, siendo el primero de estos hitos (2007) un factor asociado al impacto del movimiento estudiantil del año anterior, y del Consejo Asesor (2006) que tuvo un papel decisivo en su resolución. Podría también vincularse el crecimiento presupuestario del año 2014 a las propuestas del nuevo gobierno que buscó responder a algunas demandas del movimiento estudiantil de 2011 no resueltas por el gobierno de entonces, y que habían sido puestas en la agenda política por los movimientos sociales y estudiantiles en la campaña presidencial del 2013. Sin embargo, más allá de estas situaciones, educación 
Donoso-Díaz, Arias-Rojas y Reyes-Araya: Tendencias del financiamiento público y dilemas...

registra una expansión presupuestaria relevante referida al PIB, mayor que otros ministerios del aérea social, exponiendo la prioridad país que representa.

Los datos confirman que el presupuesto del MINEDUC es clave en el desarrollo del sistema educativo, más aún tras las leyes aprobadas en el período 2014-2017. Por ende, su diseño e implementación le confirman como área sustantiva para un análisis más detallado. Este incremento presupuestario no implica que se haya dado plena respuesta a las demandas de los movimientos estudiantiles y sociales, los sucesos de octubre de 2019 en el país dan cuenta que muchas de estas demandas siguen vigentes y se presentan como críticas respecto a las insatisfactorias reformas realizadas por todos los gobiernos, evidenciando la compatibilidad de intereses comprometidos por ambos espectros políticos de derecha y de izquierda.

En razón de ello, la pregunta sobre la necesidad de mayores recursos para el sector debe ir acompañada de otras consideraciones sobre cómo mejorar la eficiencia y eficacia del gasto, no siendo el único camino, sino también generando mayores impactos en temas sustantivos como la alta segmentación social, la vulnerabilidad de los estudiantes y la calidad de la educación en la mayor parte de sus niveles y estratos sociales.

Ello no implica que se congele la expansión del presupuesto, sino que sería razonable estudiar las brechas de gasto en referencia con los costes reales de formación de estudiantes de los distintos niveles del sistema según su situación socioeconómica, y así responder con mayores argumentos: ¿Cuán posible es mantener en el tiempo esta tasa de crecimiento del presupuesto en educación, en un país con una estructura productiva como Chile? ¿Es posible pensar en un diseño estructural presupuestario diferente de asignación de los recursos que responda a criterios de logro de metas y otros componentes más vinculados con políticas públicas estratégicas en este campo? ¿Cuán distinta debiese ser esta asignación, al fijarse parámetros de control y evaluación asociados a la calidad e inclusión, metas, rendición de cuentas o relación intrasistema entre oferta o demanda? ¿Será necesario explorar marcos presupuestarios plurianuales, pensando en políticas educativas de largo plazo más allá (o no) del período presidencial? 


\section{Agradecimientos}

Se agradece a la Agencia Nacional de Investigación y Desarrollo de Chile, proyecto FONDECYT regular 1190086.

\section{Referencias}

Asociación de Municipalidades de Chile (AMUCH). (2015). Municipalidades y Educación. Algunas aproximaciones al aporte del Sistema Municipal Chileno a la Educación Pública. AMUCH. http://www.amuch.cl/p df/municipalidad_y_educacion.pdf

Banco Central de Chile (2018). Estadísticas macroeconómicas de Producto Interno Bruto (bases de datos estadísticas macroeconómicas de períodos anuales 1999 al 2018). Estadísticas de cuentas nacionales del Banco Central de Chile.

Banco Interamericano de Desarrollo (BID). (2018). El gasto en educación: cuando cada centavo cuenta. En A. Izquierdo, C. Pessino \& G. Vuletin (eds.), Mejor gasto para mejores vidas: cómo América Latina y el Caribe puede hacer más con menos (pp. 183-213). Banco Interamericano de Desarrollo. https://publications.iadb.org/bitstream/handle/11319 19152/DIA-2018-Mejor-gasto-para-mejores-vidas.pdf?isAllowed=y \&sequence $=4$

Beyer, H. (2007). Una nota sobre financiamiento de la educación. En J. J. Brunner \& C. Peña (coords.), La Reforma al Sistema Escolar: aportes al debate (pp. 179-200). UDP.

Blöndal, J. \& Curristine, T. (2004). Budgeting in Chile. OECD Journal on Budgeting, 4(2), 7-45. https://www.oecd.org/chile/40139802.pdf

Cetrángolo, O. \& Curcio, J. (2017). Financiamiento y gasto educativo en América Latina [Serie Macroeconomía del desarrollo No. 192]. Comisión Económica para América Latina y el Caribe (CEPAL). https: //www.cepal.org/sites/default/files/publication/files/42403/S1701080 _es.pdf 
Donoso-Díaz, Arias-Rojas y Reyes-Araya: Tendencias del financiamiento público y dilemas...

Cetrángolo, O. \& Curcio, J. (2017a, 23 y 24 de marzo). Financiamiento y gasto educativo en América Latina, a partir de la última generación de reformas [ponencia]. XXIX Seminario Regional de Política Fiscal, Santiago, Chile.

Congreso Nacional de Chile. (2018, 11 mayo). Ley 21091. Sobre Educación Superior. https://www.bcn.cl/leychile/navegar?idNorma=1118991

Congreso Nacional de Chile. (2017, 16 de noviembre). Ley 21040. Crea el Sistema de Educación Pública. https://www.bcn.cl/leychile/navegar?i dNorma $=1111237$

Congreso Nacional de Chile (2016, 04 de marzo). Ley 20903. Crea el Sistema de Desarrollo Profesional Docente y modifica otras normas. https://www. bcn.cl/leychile/navegar?idNorma $=1087343$

Congreso Nacional de Chile (2015, 29 de mayo). Ley 20845. De Inclusión Escolar que regula la admisión de los y las estudiantes, elimina el financiamiento compartido y prohibe el lucro en establecimientos educacionales que reciben aportes del Estado. https://www.bcn.cl/ley chile/navegar?idNorma $=1078172$

Congreso Nacional de Chile (2012, 24 de septiembre). Ley 20630. Perfecciona la legislación tributaria y financia la reforma educacional. https://www. bcn.cl/leychile/navegar?idNorma $=1043598$

Consejo Asesor Presidencial para la Calidad de la Educación. (2006). Informe Final. Presidencia de la República. http://educacion2020.cl/documen tos/informe-final-de-consejo-asesor-presidencial-para-la-calidad-de-la -educacion/

Contraloría General de la República (CGR). (2018). Informes anuales consolidados de la Contabilidad General de la Nación, (informes anuales, período 2000-2018). División de Contabilidad y Finanzas Públicas (CGR), Chile.

Darville, P. \& Rodríguez, J. (2007). Institucionalidad, financiamiento y rendición de cuentas en educación [Serie estudios de finanzas públicas No. 
10]. Dirección del Presupuesto del Ministerio de Hacienda (DIPRES). https://www.dipres.gob.cl/598/articles-21658_doc_pdf.pdf

Dirección de Presupuestos (DIPRES). (2019). Ciclo Presupuestario. http://ww w.dipres.cl/598/w3-article-3699.html

Dirección de Presupuestos (DIPRES). (2018). Informes anuales de ejecución presupuestaria del Ministerio de Educación (informes de ejecución presupuestaria período anual 1999-2018). DIPRES, Ministerio de Hacienda.

Dirección de Presupuestos (DIPRES). (2017). Balance de Gestión Integral. Ministerio de Educación, Subsecretaría de Educación. (Balances de Gestión Integral período 2010-2017). DIPRES, Ministerio de Hacienda.

Dirección de Presupuestos (DIPRES). (2014). Estado de la Hacienda Pública 2014 (presentación del Ministro de Hacienda, Alberto Arenas de Mesa). Dirección de Presupuestos, Ministerio de Hacienda. http://biblioteca digital.dipres.gob.cl/bitstream/handle/11626/10535/EstadoHaciend aPublica2014.pdf? sequence $=1$ \&isAllowed $=y$

Gallego, F. \& Sapelli, C. (2007). El financiamiento de la educación en Chile: una evaluación. Pensamiento Educativo. Revista de Investigación Educacional Latinoamericana, 40(1), 263-284. http://ojs.uc.cl/index. $\mathrm{php/pel/article/view/25511}$

González, P. (2003). Estructura Institucional, recursos y gestión en el sistema escolar chileno. En C. Cox (ed.), Políticas educacionales en el cambio de siglo. La reforma del sistema escolar en Chile (pp. 213 -252). Editorial Universitaria.

Hanushek, E. \& Woessmann, L. (2015). The Knowledge Capital of Nations: Education and the Economics of Growth, The Mit Press.

Howlett, M. (2019). The Policy Design Primer. Choosing the Right Tools for the Job. Routledge Textbooks in Policy Studies. 
Donoso-Díaz, Arias-Rojas y Reyes-Araya: Tendencias del financiamiento público y dilemas...

Kremerman, M. (2009). Radiografía del financiamiento de la educación chilena: diagnóstico, análisis y propuestas. Observatorio Chileno de Políticas Educativas. http://www.opech.cl/Libros/doc3.pdf

Marcel, M. (2009). ¿Por qué la Educación Municipal? En M. Marcel, \& D. Raczynski, (eds.), La asignatura pendiente. Claves para la revalidación de la educación pública de gestión local en Chile (pp. 33-46). Uqbar Editores.

Marcel, M. \& Tokman, C. (2005). ¿Cómo se financia la educación en Chile? [Serie de Estudios de Finanzas Públicas, 5]. Dirección de Presupuestos, Ministerio de Hacienda. https://www.dipres.gob.cl/598/articles-216 69_doc_pdf.pdf

Ministerio de Educación (MINEDUC). (2018). Indicadores de la Educación en Chile, 2010-2016. Centro de Estudios, División de Planificación y Presupuesto. https://centroestudios.mineduc.cl/wp-content/uploads/s ites/100/2018/03/INDICADORES_baja.pdf

OCDE. (2019). PISA 2018 Results (Volume I): What Students Know and Can Do. OECD Publishing. https://doi.org/10.1787/5f07c754-en

OCDE. (2018). Chile. En OCDE., Education at a Glance 2018: OECD Indicators. OECD Publishing. https://doi.org/10.1787/kpiu8i-es

OCDE. (2017). Educación en Chile. Evaluación de Politicas nacionales de Educación. OCDE y Fundación SM. http://archivos.agenciaeducac ion.cl/Educacion_en_Chile_OCDE_Nov2017.pdf

OCDE. (2017a). OCDE Revisiones de recursos escolares: Chile 2017 [informe de revisión]. OECD- Ministerio de Educación. http://www.oecd.org/c hile/oecd-reviews-of-school-resources-chile-2017-9789264287112-es. htm

Orellana, V. (2018). A modo de epílogo. En V. Orellana (ed.), Entre el mercado gratuito y la educación pública. Dilemas de la educación chilena actual. LOM.

Programa de las Naciones Unidas para el Desarrollo (PNUD). (2017). Desiguales. Orígenes, cambios y desafíos de la brecha Social en Chile. 
PNUD. https://www.cl.undp.org/content/chile/es/home/library/ poverty/desiguales--origenes--cambios-y-desafios-de-la-brecha-social -en-.html

Raczynski, D. \& Salinas, D. (2009). Prioridades, actores y procesos en la gestión municipal de la educación. En M. Marcel M. \& D. Raczynski (eds.), La asignatura pendiente. Claves para la Revalidación de la Educación Pública de Gestión Local en Chile. Uqbar Editores.

Rodríguez, J., Chamorro, J., \& Vega, A. (2016). Principales tendencias del gasto fiscal en el periodo 2001-2015 [Serie de Estudios de Finanzas Públicas]. Dirección de Presupuestos, Ministerio de Hacienda. http: //www.dipres.gob.cl/598/articles-147065_doc_pdf.pdf

Romaguera, P. \& Gallegos, S. (2011). Financiamiento de la educación en Chile: desafíos de calidad y equidad. En A. Bárcena \& O. Kacef (comps.), La politica fiscal para el afianzamiento de las democracias en América Latina. Reflexiones a partir de una serie de estudios de caso (pp. 159-183). https://repositorio.cepal.org/handle/11362/4181

Treviño, E. (2018). Diagnóstico del sistema escolar: las reformas educativas 2014-2017. En I. Sánchez, (ed.), Ideas en educación II, definiciones en Tiempos de Cambio (pp. 133-166). Ediciones Universidad Católica.

UNESCO (2013). Situación Educativa de América Latina y el Caribe: Hacia la educación de calidad para todos al 2015. Oficina Regional de Educación para América Latina y el Caribe (OREALC/UNESCO). http://www. unesco.org/new/fileadmin/MULTIMEDIA/FIELD/Santiago/images/ SITIED-espanol.pdf

Villarroel, L. (2013). Financiamiento y Desfinanciamiento en la Educación Municipal. Causas, variables que inciden y propuestas. CHILEDUC. http: //educacion2020.cl/sites/default/files/financiamiento_y_desfinanciami ento_en_la_educacion_municipal.pdf

Von Gersdorff, H. (2018). El proceso presupuestario en Chile: opciones de reforma. En I. Aninat, \& S. Razmilic (eds.), Un Estado para la Ciudadania. Estudios para su modernización (pp. 533-574). Colección 
Donoso-Díaz, Arias-Rojas y Reyes-Araya: Tendencias del financiamiento público y dilemas...

Centro de Estudios Públicos- CEP. https://www.cepchile.cl/cep/site/d ocs/20180416/20180416105800/libro_un_estado_para_la_ciudadani a_vogersdorff.pdf 